This is the submitted version of the article:

Shu L., Ke S., Fei L., Huang W., Wang Z., Gong J., J iang X., Wang L., Li F., Lei S., Rao Z., Zhou Y., Zheng R.-K., Yao X., Wang Y., Stengel M., Catalan G.. Photoflexoelectric effect in halide perovskites. Nature Materials, (2020). . : - . 10.1038/s41563-020-0659-y.

Available at: https://dx.doi.org/10.1038/s41563-020-0659-y 


\section{Photoflexoelectric effect in halide perovskites}

Longlong Shu ${ }^{1 a^{*}}$, Shanming Ke ${ }^{1^{*}}$, Linfeng Fei ${ }^{1^{*}}$, Wenbin Huang ${ }^{2^{*}}$, Zhiguo Wang ${ }^{2}$, Jinhui Gong ${ }^{1}$, Xiaoning Jiang ${ }^{3}$, Li Wang ${ }^{1}$, Fei Li ${ }^{4}$, Shuijin Lei $^{1}$, Zhenggang Rao ${ }^{1}$, Yangbo Zhou ${ }^{1}$, Renkui Zheng ${ }^{1}$, Xi Yao ${ }^{4}$, Yu Wang ${ }^{1}$, Massimiliano Stengel ${ }^{5,6}$, Gustau Catalan ${ }^{5,7 b}$

${ }^{1}$ School of Materials Science and Engineering, Nanchang University, Nanchang 330031, P. R. China

${ }^{2}$ The State Key Lab of Mechanical Transmissions, Chongqing University, Chongqing 400044, P. R. China

${ }^{3}$ Department of Mechanical and Aerospace Engineering, North Carolina State University, Raleigh, NC 27695, USA

${ }^{4}$ Electronic Materials Research Laboratory, Key Laboratory of the Ministry of Education and International Center for Dielectric Research, Xi'an Jiao Tong University, Xi'an 710049, P. R. China

5Institucio Catalana de Recerca i Estudis Avançats (ICREA), Barcelona, Catalonia.

${ }^{6}$ Institut de Ciencia de Materials de Barcelona (ICMAB), CSIC, Campus Universitat Autonoma de Barcelona, Barcelona, Catalonia.

${ }^{7}$ Institut Catala de Nanociencia i Nanotecnologia (ICN2), CSIC-BIST, Campus Universitat Autonoma de Barcelona, Bellaterra 08193, Barcelona, Catalonia.

Harvesting environmental energy to generate electricity is a key scientific and technological endeavor of our time. Photovoltaic conversion and electromechanical transduction are two common energy-harvesting mechanisms based on, respectively, semiconducting junctions and piezoelectric insulators. However the different material families on which these transduction phenomena are based complicate their integration into single devices. Here, we demonstrate that halide perovskites, a family of highly efficient photovoltaic materials [1-3], display a photoflexoelectric effect whereby, under illumination, they generate the highest bendinginduced polarization so far reported for any material. We also show that photoflexoelectricity is not exclusive to halides but a general property of semiconductors that potentially enables simultaneous electromechanical and photovoltaic transduction and harvesting in unison from multiple energy inputs.

*These authors contributed equally to this work.

a Corresponding email: Ilshu@ncu.edu.cn

b Corresponding email: gustau.catalan@icn2.cat 
Flexoelectricity is a property that allows dielectric materials of any symmetry to convert inhomogeneous deformations, such as bending, into electricity (4-7). Because intrinsic flexoelectric coefficients tend to be small (7), large deformations are required in order to achieve substantial flexoelectric responses; hence, the most dramatic manifestations of flexoelectricity tend to be found at the nanoscale, where large gradients are easier to achieve (8-11). However, the discovery of a large flexoelectric-like response in bulk semiconductors[12], has altered this landscape, empowering flexoelectricity at the macroscale and setting the scene for combining flexoelectricity with semiconductor physics. In this context, a flexophotovoltaic effect (13) has already been demonstrated whereby strain gradients enhance photovoltaic performance, and it is therefore natural to wonder whether there is a converse 'photoflexoelectric effect' whereby light absorption can be used to enhance flexoelectric response. Here we show that semiconductor photoflexoelectricity does exist and can be large: halide perovskites under light yield effective flexoelectric coefficients orders of magnitude larger than in the dark, and millions of times bigger than the conventional flexoelectricity of dielectrics.

Halide perovskites, also known as hybrid organic-inorganic perovskites, of which $\mathrm{MAPbl}_{3}$ (MA refers to methylammonium cation, $\mathrm{CH}_{3} \mathrm{NH}_{3}{ }^{+}$) is the archetype, have surged in the global pursuit of third-generation solar cells due to their vertiginous escalation up the photovoltaic efficiency tables (1-3); despite their recent discovery, the efficiency of $\mathrm{MAPb}_{3}$-based solar cells already exceeds $20 \%$, a benchmark that took decades for silicon to reach. Halide perovskites have yet to overcome practical problems such as photo-degradation (14), but this has not eclipsed their appeal. Here we have measured the flexoelectric coefficient of single crystals of $\mathrm{CH}_{3} \mathrm{NH}_{3} \mathrm{PbBr}_{3}$ and $\mathrm{CH}_{3} \mathrm{NH}_{3} \mathrm{PbCl}_{3}$, hereafter $\mathrm{MAPbBr}$ and $\mathrm{MAPbCl}$. We targeted these compositions instead of the archetypal iodide composition, $\mathrm{MAPbl}_{3}$, because the latter is thought to be ferroelectric (15), whereas chloride and bromide compositions are centrosymmetric at room temperature (16), which excludes piezoelectricity and facilitates the flexoelectric analysis. Two of our samples are pictured in the inset of Figure 1a. The fabrication procedure is described in the Methods section (see also Figure S1), and X-ray diffraction confirms single-orientation with $\{100\}$ planes parallel to the surfaces and no detectable crystalline impurities (Figure S2).

In order to measure flexoelectricity, we use a piezoelectric actuator to deliver an oscillatory force to the free end of clamped cantilever-shaped crystals with a capacitor structure (electrode/halide/electrode). The oscillatory bending induces an alternating current that is measured with a lock-in amplifier connected to the electrodes with platinum wires. The strain gradient is calculated from the deflection amplitude using the standard elastic deformation equations for a point-loaded single-clamp beam, and the polarization is extracted from the amplitude of the oscillating current (see Methods). The linear proportionality between polarization and strain gradient is shown in Figure 1-a; the slope of this proportionality is the flexoelectric coefficient.

The effective flexoelectric coefficients, $\mu_{\text {eff, }}$ are quite stable as a function of temperature (Figure 1-b) and of the order of $\sim 30 \mu \mathrm{C} / \mathrm{m}$ for both halide compositions. Given their similarity, hereafter we show only results for $\mathrm{MAPBr}$, and the results for $\mathrm{MAPCl}$ are provided in the Supplementary Materials. These flexoelectric coefficients are large, comparable with those of the best electroceramics [7]. We have verified that their origin is not piezoelectric by performing uniaxial compression measurements (Figure 1-c). We additionally notice that the effective flexoelectric coefficients are influenced by the type of electrode and proportional to thickness (Figure 1-d), consistent with barrier-layer flexoelectricity in semiconductors [12]. The capacitance and dielectric losses also behave as expected from a barrier-layer capacitor (Supplementary Figure S3). 

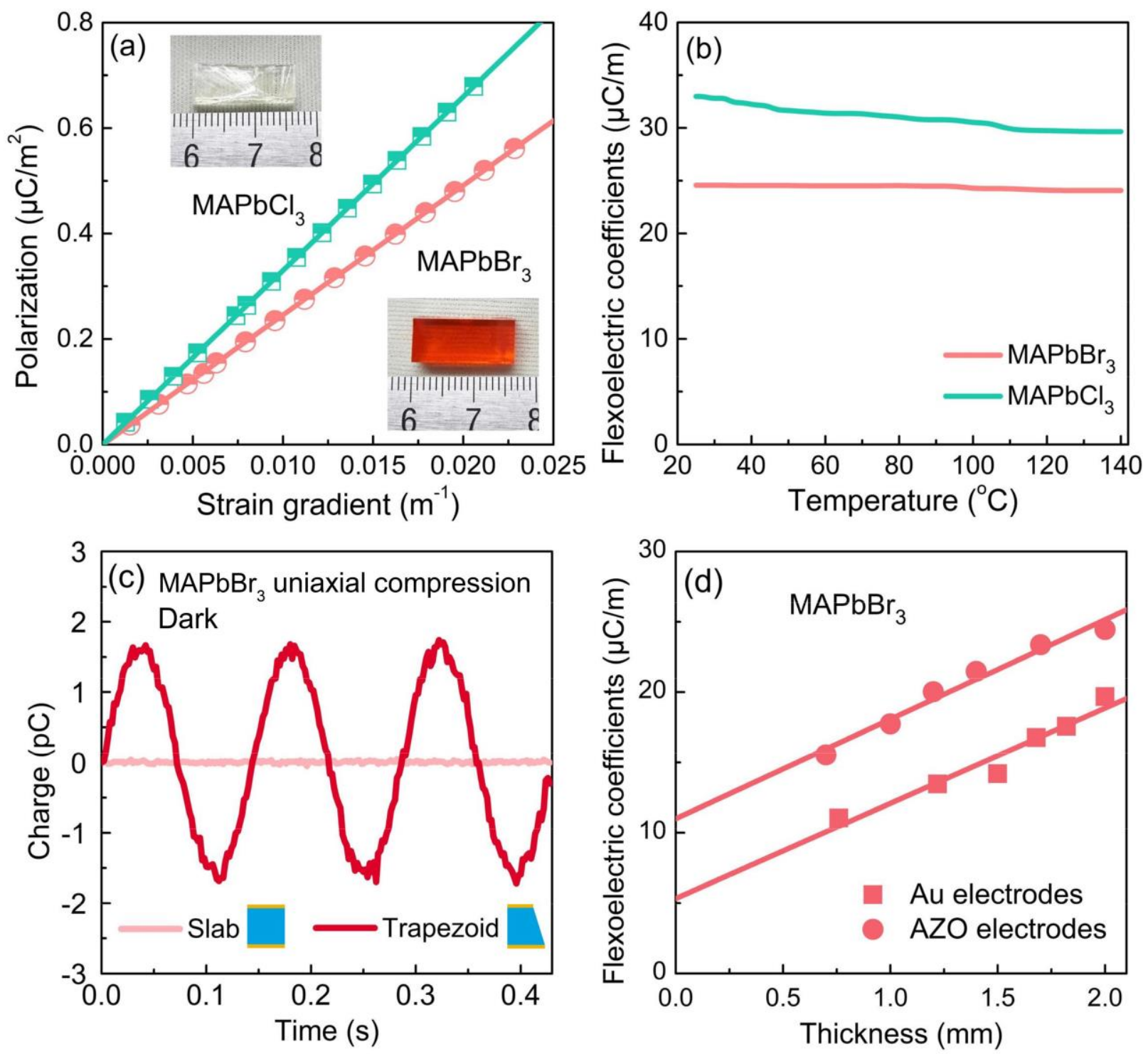

Figure 1 Flexoelectricity of perovskites in the dark. (a) Electric polarization vs curvature for two crystals and linear least-squares fit to the results. The slope yields the effective flexoelectric coefficients. Inset: photographs of single crystal samples of the two compositions examined (b) Temperature dependence of the flexoelectric coefficient of halide perovskites in the dark (c) Comparison between uniaxial compression of a slab-shaped sample (parallel walls) and a trapezoid-shaped sample. The uniaxial force was $60 \mathrm{~N}$. The vertical asymmetry of the trapezoid yields a longitudinal train gradient that generates a flexoelectric charge, whereas slab compression yields no polarization under compression, confirming that it is not piezoelectric. (d) Flexoelectric coefficient as a function of thickness for $\mathrm{MAPbBr}_{3}$ crystals with two different types of electrode, Au and transparent oxide AZO.

For experiments under illumination, we used transparent electrodes based on $\mathrm{AZO}\left(98 \% \mathrm{ZnO}+2 \% \mathrm{Al}_{2} \mathrm{O}_{3}\right.$, see Methods). The electrodes' transmission spectra and halides' absorption spectra were measured to determine the optimal wavelength range for electrode transparency and halide absorption (Supplementary Figure S4). We then measured the flexoelectricity under illumination. A scheme of the experimental concept is shown in Figures 2(a-b), and pictures of the actual experimental setup are shown in the Supplementary Materials (Figure 
S5). The applied strain gradient and measured displacement current as a function of time are plotted in Figure 2-c and 2-d respectively.

(a)

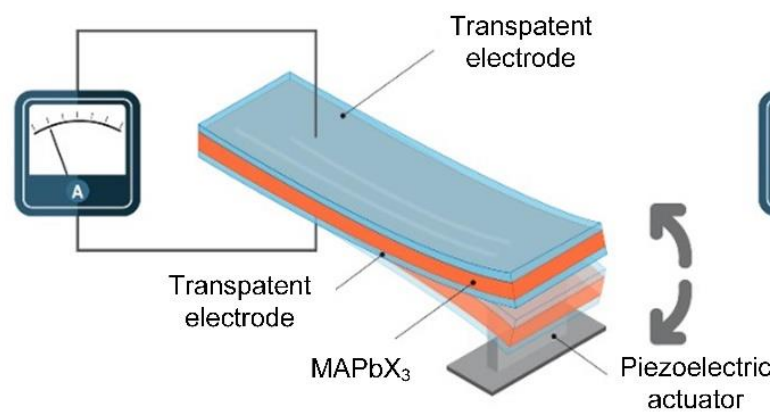

(b)

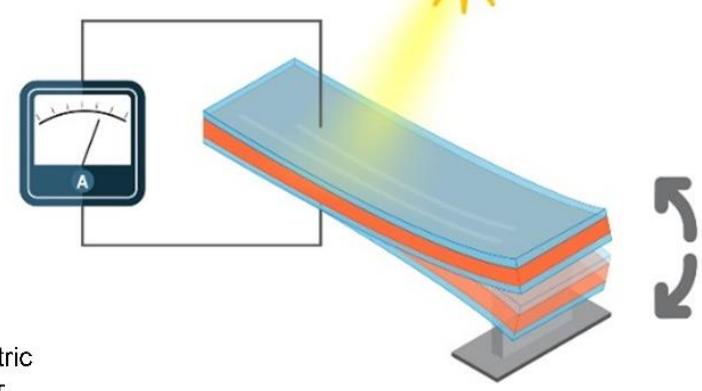

(c)

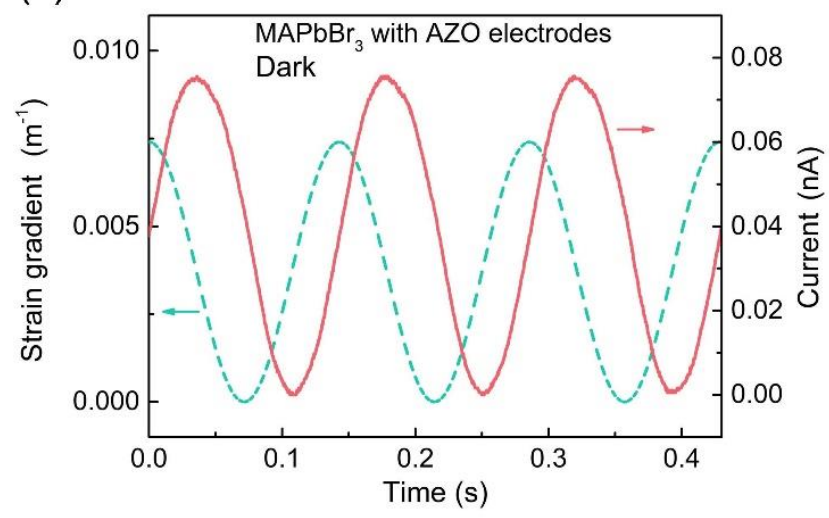

(d)

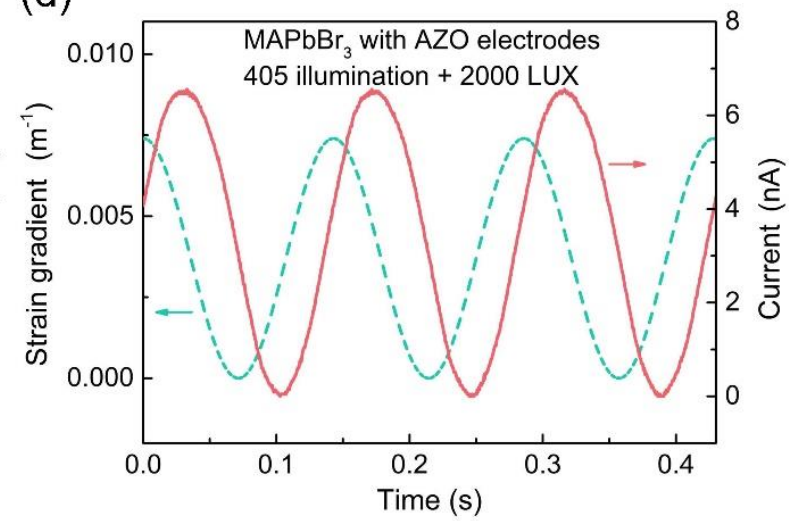

Figure 2. Photoflexoelectric experiment. A piezoelectric actuator is used to bend in an oscillatory manner the cantilever-shaped halide capacitor with transparent electrodes in the dark (a) or under light (b). Displacement current and strain gradient as a function of time in the dark (c) and with light (d). The current is 90 degrees outof-phase with the strain gradient and is therefore a displacement current.

The flexoelectric coefficients under different light sources are summarized in Table 1 . The results are consistent with the optical absorption spectra: photoflexoelectric enhancement happens only when the light is absorbed by the halide, i.e., when its wavelength is smaller than the bandgap. At the same time, $365 \mathrm{~nm}$ light is less efficient than $405 \mathrm{~nm}$ due to the lower transparency of the electrodes.

Table 1: Halides' photonic absorption edge bandgap and flexoelectric coefficients as a function of wavelength.

\begin{tabular}{lllllll}
\hline \hline Material & Absorption edge & $\begin{array}{l}365 \mathrm{~nm} \\
(2000 \mathrm{LUX})\end{array}$ & $\begin{array}{l}405 \mathrm{~nm} \\
(2000 \mathrm{LUX})\end{array}$ & $\begin{array}{l}532 \mathrm{~nm} \\
(4200 \mathrm{LUX})\end{array}$ & $\begin{array}{l}\text { 650nm } \\
(3800 \mathrm{LUX})\end{array}$ & dark \\
\hline $\mathrm{MAPbBr}_{3}$ & $574 \mathrm{~nm}$ & $1705 \mu \mathrm{C} / \mathrm{m}$ & $2078 \mu \mathrm{C} / \mathrm{m}$ & $1080 \mu \mathrm{C} / \mathrm{m}$ & $24 \mu \mathrm{C} / \mathrm{m}$ & $24 \mu \mathrm{C} / \mathrm{m}$ \\
$\mathrm{MAPbCl}_{3}$ & $431 \mathrm{~nm}$ & $1880 \mu \mathrm{C} / \mathrm{m}$ & $2185 \mu \mathrm{C} / \mathrm{m}$ & $33 \mu \mathrm{C} / \mathrm{m}$ & $33 \mu \mathrm{C} / \mathrm{m}$ & $33 \mu \mathrm{C} / \mathrm{m}$ \\
\hline \hline
\end{tabular}


Since these photo-enhanced flexoelectric coefficients are the largest reported for any material, it is important to exclude artifacts. The maximum bending amplitude in these measurements was 1 micron; for samples of $\sim 10 \mathrm{~mm}$ length, this translates into oscillations of $\sim 0.01 \%$ in optical cross-section. Geometric self-shadowing is thus negligible. Photo-electrochemical effects were also discarded by examining samples with gold electrodes, as gold is an unreactive noble metal. The flexoelectric coefficients were measured with sideillumination in order to get around the opacity of the gold. The results for gold were of the same order of magnitude as those measured with transparent AZO electrodes ( $\mu_{\text {eff }}^{\text {gold }} \approx 1500 \mu \mathrm{C} / \mathrm{m}$, see supplementary Figure S6). Uniaxial compression of symmetric and asymmetric samples (slab vs trapezoid) also shows that the effect requires gradients and is not piezoelectric. When crystals are shaped asymmetrically (e.g. in a trapezoid shape) the stress difference between top and bottom establishes a vertical strain gradient that can yield longitudinal flexoelectric polarization [7,17], and we verified that this is the case for our crystals (Supplementary Figure S7S8); in contrast, compressing parallel-sided slabs yields no charge (Figure 1-c).

Moreover, the polarization is linearly proportional to strain gradient, both in the dark (Figure 1-a) and with light (Supplementary Figure S9). Notice also that there is phase shift of 90 degrees between the bending and the current (figure $2 \mathrm{c}-\mathrm{d}$ ). This means that the photoflexoelectric response is not resistive (hence it is not a shift current (13)) but dielectric and thus originating from polar displacement: if the current is 90 degrees out of phase with the strain gradient, the polarization (from which the current is the time-derivative) is in-phase with the strain gradient. All experimental indicators are therefore consistent with a true flexoelectric effect.

Bending-induced polarization can be caused by bulk flexoelectricity (1-7), surface piezoelectricity $(6,7,18)$, residual/macroscopic piezoelectricity $(19,20)$, flexoionics (21), or semiconductor barrier polarization (12). Bulk flexoelectricity (and surface piezoelectricity) are proportional to dielectric constant $(6,7)$, which is of the order of 100-1000 for the halide crystals (Figure S3). This is comparable to the dielectric constant of oxides such as $\mathrm{SrTiO}_{3}$ (7) or $\mathrm{PbZrO}_{3}(22)$, yet the halide's flexoelectric coefficient is $\sim 10,000$ larger. In fact, the so-called flexocoupling coefficient (flexoelectric coefficient divided by the dielectric permittivity) is $>1000 \mathrm{~V}$ (Supplementary Figure S10), much larger than the theoretical upper limit of $\sim 10 \mathrm{~V}(6,7)$. Bulk flexoelectricity therefore seems unlikely to be the dominant effect.

The flexoelectric coefficient of halides (in the dark) is comparable to that of some ferroelectrics (6), but the compositions in this study are not piezoelectric. Defect gradients can introduce macroscopic piezoelectricity in non-polar materials $(19,20)$, but estimates of this contribution towards flexoelectricity are in the range of $\mathrm{nC} / \mathrm{m}$ (20), too small to explain the $\mu \mathrm{C} / \mathrm{m}$ measured in the dark and negligible compared to the $\mathrm{mC} / \mathrm{m}$ measured under light. Moreover, the slab-compression experiment shows no piezoelectricity either in the dark (Figure 1c) or with light (Figure S8). Macroscopic piezoelectricity is therefore also discarded as the source of flexoelectricity.

Halide perovskites are ionic conductors $(23,24,25)$, so they might display "flexoionic" effects (21). Our samples are single-compound single-crystals, precluding photo-induced compositional segregation (24) or grain boundary transport $(23,25)$; nevertheless, the flexoelectric response shows a weak frequency dependence in the dark (Figure 3-a). This is not a relaxation but an increase with frequency (Figure 3-a). It is consistent with the presence of a slow (ionic) contribution in addition to a frequency-independent contribution of bigger magnitude and opposite sign. Under illumination, the enhanced coefficient is independent of frequency (Figure 3-a), indicating that the faster effect is the dominant one.

Electrode and thickness-dependence (figure 1-d) suggest a semiconductor barrier-layer origin [12]. Halides have a large capacitance in the presence of light (26), which is also consistent with their barrier layer capacitor behavior (Supplementary Figure S3). The existence of photo-induced Schottky barriers is confirmed by the 
double-diode-like transport behaviour (Figure 3-b and S11-S13). (Parenthetically, we note that back-to-back Schottky diodes can display "fake" ferroelectric-like hysteresis loops (27), which may be relevant regarding the controversy about the ferroelectricity of $\mathrm{MAPbl}_{3}$ ). Photoexcitation increases free carrier concentration and decreases depletion width at the interface with the electrodes, and the photo-enhanced flexoelectricity can be related to this photo-modulation of the Schottky barriers.
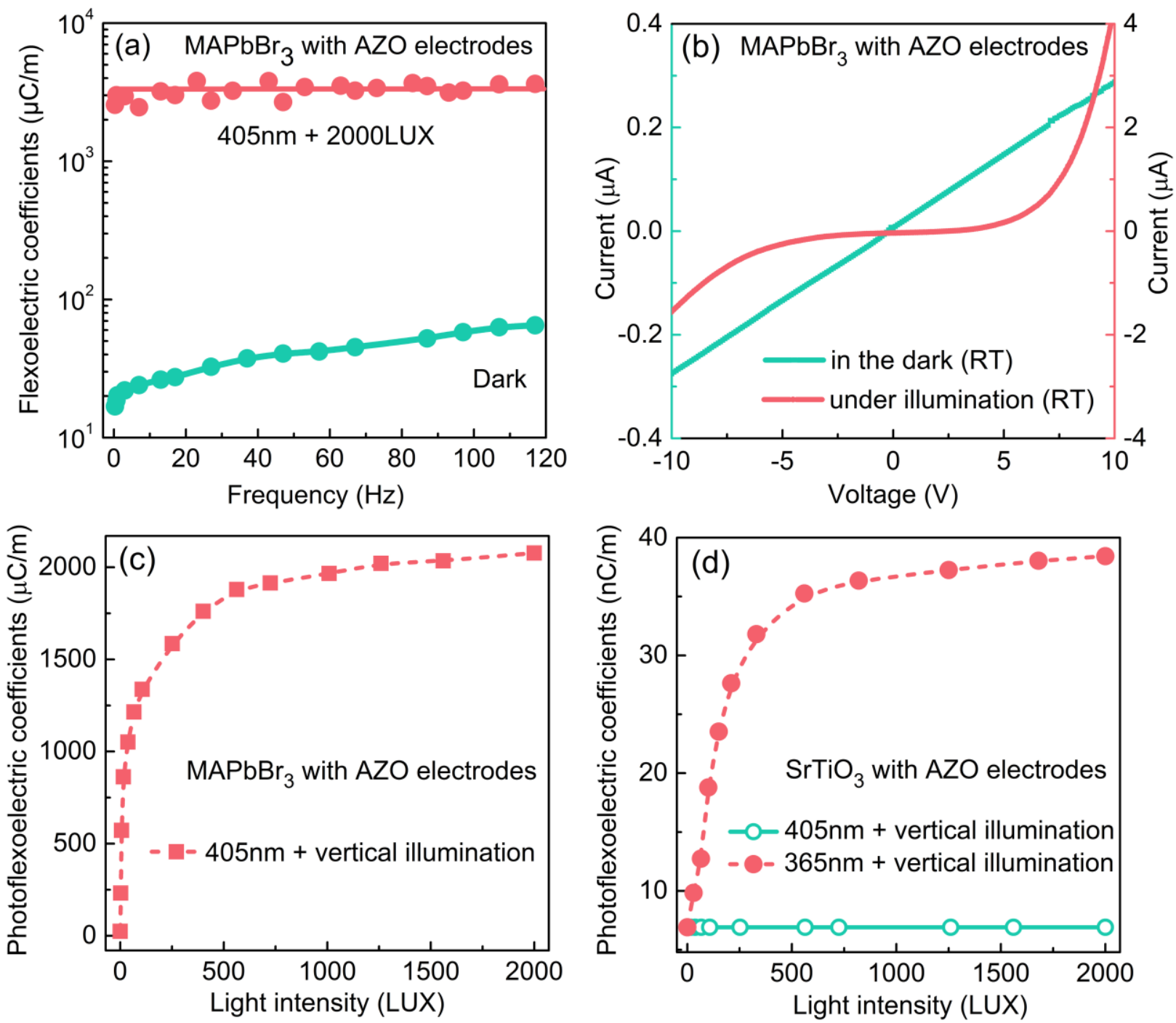

Figure 3 (a) Flexoelectric coefficient as a function of mechanical oscillation frequency. The coefficients are slightly frequency-dependent in the dark, suggesting an ionic transport contribution, but frequencyindependent under illumination, consistent with a faster electronic/dipolar process. (b) Electronic transport, showing the onset of diode-like response under illumination, indicating the formation of interfacial barrier layers due to the increased density of free carriers. (c) flexoelectric coefficient of $\mathrm{MAPBr}_{3}$ as a function of illumination and (d) flexoelectric coefficient of $\mathrm{SrTiO}_{3}$ as a function of illumination, showing that the photoflexoelectric enhancement is a general phenomenon not exclusive of halide perovskites. 
The depletion width, $d$, of a Schottky barrier, is (28):

$$
d=\sqrt{\frac{2 \varepsilon_{0} \varepsilon_{r} \phi_{0}}{n}}
$$

where $\varepsilon_{\mathrm{r}}$ is the relative dielectric constant, $n$ the density of free carriers, and $\phi_{0}$ is the Schottky barrier height measured in $\mathrm{eV}$. Note that the device is overall charge-neutral: at the metallic surface, a free carrier density $\sigma=n d$ compensates for the aforementioned depleted carriers.

The carrier density $n$-and hence the barrier width- can be modified by photoexcitation in any semiconductor, and also by piezoelectricity if the material is piezoelectric [29]. In addition, surface piezoelectricity (and even pyroelectricity) can exist in any material even if its bulk is centrosymmetric [30, 30]. If strain is applied homogeneously across the crystal, the surface-piezoelectric charges generated at both sides of the crystal are identical, generating no current between the electrodes. If there is bending, however, the strains on opposite sides of the crystal are opposite (compression in the concave side, tension in the convex one) and thus generate polarizations of opposite sign [6]. A compensating current will therefore appear in response to the bending if the electrodes on the opposite sides are connected. This bending-induced current is what we measure as flexoelectricity: the effective flexoelectric coefficient, $\mu_{e f f}$, is defined as the change in surface charge density divided by the strain gradient.

The surface strain caused by bending is geometrically given by $\eta=\frac{t}{2} G$, where $t$ is crystal thickness and $G$ is curvature ( $G \equiv$ strain gradient). The surface-strain-induced modification of the interfacial charge density is captured by the surface deformation potential (18): the change in barrier height due to surface strain, $\varphi \equiv \frac{\partial \phi}{\partial \eta}$. The effective flexoelectric coefficient of a bent semiconductor with interfacial barrier layers is, therefore:

$$
\mu_{e f f} \equiv \frac{\partial \sigma}{\partial G}=\frac{\partial(n d)}{\partial \phi} \frac{\partial \phi}{\partial G}=\sqrt{\frac{n \varepsilon_{0} \varepsilon_{r}}{2 \phi_{0}}} \varphi \frac{t}{2}
$$

Eq (2) reproduces qualitatively all the features of the photoflexoelectric effect. In particular, the effective flexoelectric coefficient is proportional to sample thickness; it depends on the semiconductor-electrode interface via the Schottky barrier height, $\phi_{0}$; and it grows non-linearly as with the carrier density $n$ and thus also with the photo-exciting illuminance, as observed (figure 3-c). Photoflexoelectricity should therefore be a general property of semiconductors and not exclusive of halide perovskites. To illustrate this point, we have also measured it in $\mathrm{SrTiO}_{3}$ under UV light (Figure 3-d).

For halides, the "photoflexoelectric coefficient", defined as $\gamma \equiv \mu_{\text {light }}-\mu_{\text {dark }} / \mu_{\text {dark }}$ is enormous, reaching $10000 \%$. As a consequence, the photo-enhanced effective flexoelectric coefficients of halide perovskites are the highest on record (see comparative chart in Figure 4). By comparison, the equivalent effect in liquid crystals is less than $10 \%(32)$. 


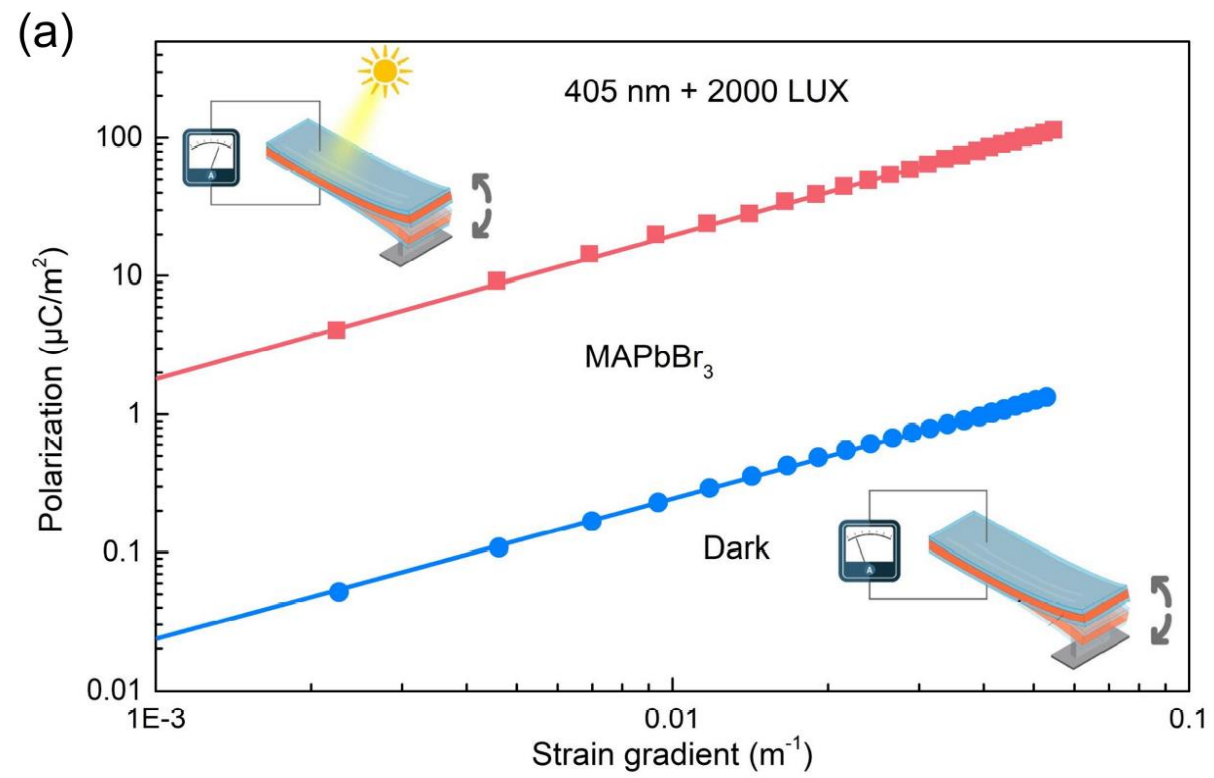

(b)

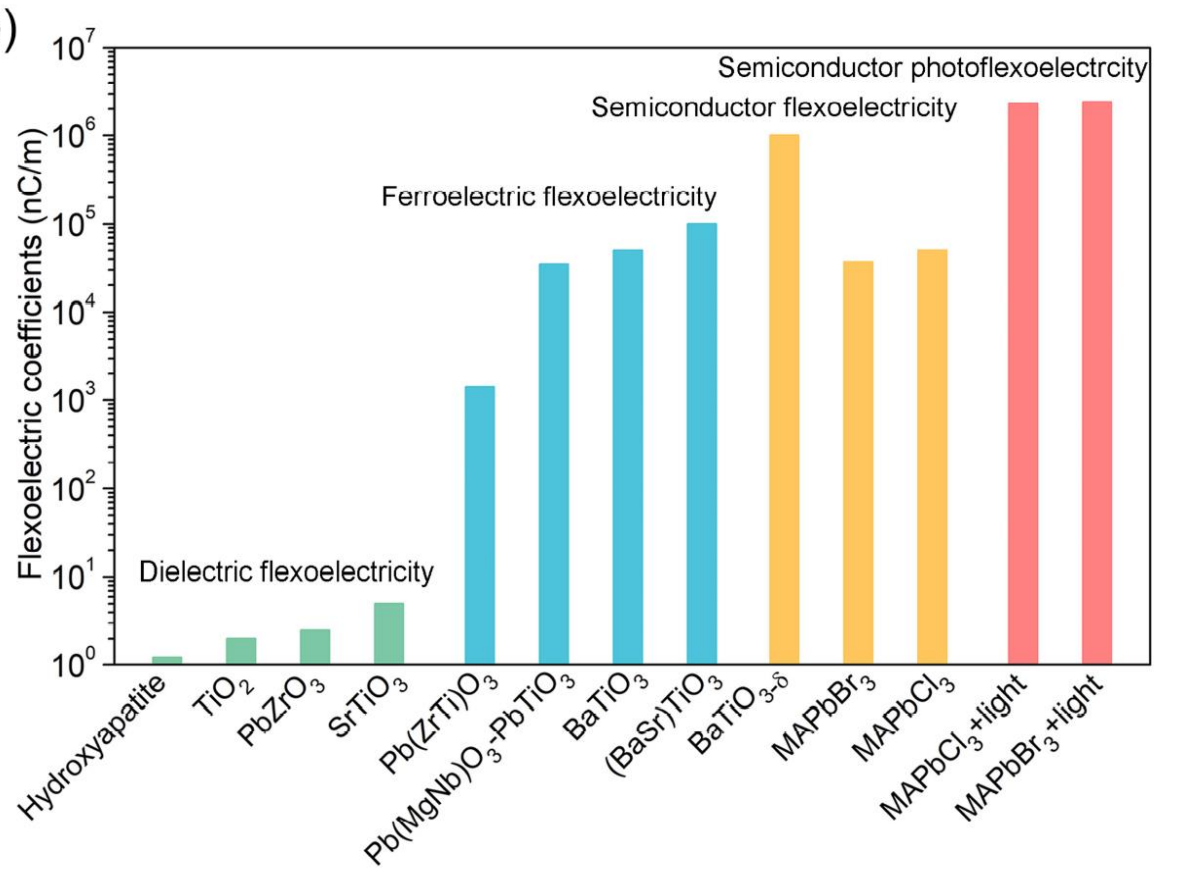

Figure 4: (a) Comparison between the bending-induced polarization of $\mathrm{MAPB}_{3}$ in the dark and with light. (b) Comparison of effective flexoelectric coefficients for different families of materials. The physical mechanism is qualitatively different for each family: bulk flexoelectricity in dielectrics, residual piezoelectricity in ferroelectrics, and barrier layer polarization in semiconductors.

Light and strain gradients thus work together to enable photoflexoelectric devices with a performance above that of flexoelectricity alone. The ability of photoflexoelectricity to combine energy inputs from light and vibrations suggests a use in photo-electromechanical multi-harvesters [33] that can still supply energy in the absence of one input, or yield a "bumper harvest" in the presence of both. 


\section{REFERENCES}

1. A. Kojima et al., Organometal halide perovskites as visible-light sensitizers for photovoltaic cells. J. Am. Chem. Soc. 131, 6050-6051 (2009).

2. J. Burschka et al., Sequential deposition as a route to high-performance perovskite-sensitized solar cells. Nature 499, 316-319 (2013).

3. W. Li et al., Chemically diverse and multifunctional hybrid organic-inorganic perovskites. Nat. Rev. Mater. 2, 16099 (2017).

4. S. M. Kogan, Piezoelectric effect during inhomogeneous deformation and acoustic scattering of carriers in crystals. Sov. Phys. Solid State 5, 2069-2070 (1964).

5. E. Bursian, O. I. Zaikovskii, Changes in curvature of ferroelectric film due to polarization. Sov. Phys. Solid State 10, 1121-1124 (1968).

6. A. K. Tagantsev, A. K. Piezoelectricity and flexoelectricity in crystalline dielectrics. Phys. Rev. B 34, 5883-5889 (1986).

7. P. Zubko, G. Catalan, A. K. Tagantsev, Flexoelectric effect in solids. Annu. Rev. Mater. Res. 43, 387-421 (2013).

8. M. S. Majdoub, P. Sharma, T. Cagin, Enhanced size-dependent piezoelectricity and elasticity in nanostructures due to the flexoelectric effect. Phys. Rev. B 77, 125424 (2008).

9. D. Lee et al., Giant flexoelectric effect in ferroelectric epitaxial thin films. Phys. Rev. Lett. 107, 057602 (2011).

10. H. Lu et al., Mechanical writing of ferroelectric polarization. Science 336, 59-61 (2012).

11. U. K. Bhaskar et al., A flexoelectric microelectromechanical system on silicon. Nat. Nanotechnol. 11, 263-266 (2016).

12. J. Narvaez, F. Vasquez-Sancho, G. Catalan. Enhanced flexoelectric-like response in oxide semiconductors. Nature 538, 219-221 (2016).

13. M.-M. Yang, D. J. Kim, M. Alexe, Flexo-photovoltaic effect. Science 360, 904-907 (2018).

14. W. Nie et al., Light-activated photocurrent degradation and self-healing in perovskite solar cells, Nat. Commun. 7, 11574 (2016).

15. Y. Rakita et al., Tetragonal CH3NH3Pbl3 is ferroelectric, Proc. Natl. Acad. Sci. 114, E5504 - E5512 (2017).

16. Y. Liu et al., Two-inch-sized perovskite $\mathrm{CH}_{3} \mathrm{NH}_{3} \mathrm{PbX}_{3}(\mathrm{X}=\mathrm{Cl}, \mathrm{Br}, \mathrm{I})$ crystals: growth and characterization. Adv. Mater. 27, 5176-5183 (2015).

17. W. Zhu, J. Y. Fu, N. Li, and L. Cross, Piezoelectric composite based on the enhanced flexoelectric effects. Appl. Phys. Lett. 89, 192904 (2006).

18. M. Stengel, Surface control of flexoelectricity. Phys. Rev. B 90, 201112 (2014).

19. A. Biancoli, C. M. Fancher, J. L. Jones, D. Damjanovic, Breaking of macroscopic centric symmetry in paraelectric phases of ferroelectric materials and implications for flexoelectricity. Nat. Mater. 14, 224229 (2015).

20. A. Abdollahi, F. Vásquez-Sancho, G. Catalan, Piezoelectric mimicry of flexoelectricity. Phys. Rev. Lett. 121, 205502 (2018).

21. X. Wen et al., Flexoelectret: An Electret with a Tunable Flexoelectriclike Response. Phys. Rev. Lett. 122, 148001 (2019).

22. P. Vales-Castro et al., Flexoelectricity in antiferroelectrics, Appl. Phys. Lett. 113, 132903 (2018).

23. Z. Xiao et al., Giant switchable photovoltaic effect in organometal trihalide perovskite devices. Nat. Mater. 14, 193 - 198 (2015). 
24. E. T. Hoke et al., Reversible photo-induced trap formation in mixed-halide hybrid perovskites for photovoltaics. Chem. Sci. 6, $613-617$ (2015).

25. J. Xing et al., Ultrafast ion migration in hybrid perovskite polycrystalline thin films under light and suppression in single crystals, Phys. Chem. Chem. Phys. 18, 30484-30490 (2016).

26. E. J. Juarez-Perez et al., Photoinduced giant dielectric constant in lead halide perovskite solar cells. J. Phys. Chem. Lett. 5, 2390-2394 (2014).

27. L. Pintilie, M. Alexe, Ferroelectric-like hysteresis loop in nonferroelectric systems. Applied Physics Letters 87, 112903 (2005).

28. R. F. Pierret, Semiconductor Device Fundamentals (Addison-Wesley, Reading, MA, 1996), 213-214.

29. Y. Liu , Q. Yang , Y. Zhang , Z. Yang , and Z.L. Wang, Nanowire Piezo-phototronic Photodetector: Theory and Experimental Design, Adv.Mater. 24, 1410-1417 (2012).

30. E, Meirzadeh, D. V. Christensen, E. Makagon, H. Cohen, I. Rosenhek - Goldian, E. H. Morales, A. Bhowmik J. M. G. Lastra, A. M. Rappe, D. Ehre, M. Lahav, N. Pryds, Igor Lubomirsky, Surface Pyroelectricity in Cubic $\mathrm{SrTiO}_{3}$. Adv. Mat. 31, 1904733 (2019).

31. G. Catalan, B. Noheda, Surface polarization feels the heat. Nature 575, 601 (2019).

32. Y. Marinov et al., Photoflexoelectric effects in a homeotropic guest-host nematic. Europhys. Lett. 41, 513-518 (1998).

33. A. S. Weddell et al. A survey of multi-source energy harvesting systems. Proc. Design Autom. Test Eur. Conf. Exhibit. 905-908 (2013).

Requests for additional materials can be made to Longlong Shu, llshu@ncu.edu.cn. Questions can be addressed to Longlong Shu or Gustau Catalan, gustau.catalan@icn2.cat.

\section{METHODS}

\section{Single crystal preparation}

The $\mathrm{CH}_{3} \mathrm{NH}_{3} \mathrm{PbX}_{3}\left(\mathrm{MAPbX}_{3}\right)$ perovskite single crystals were prepared using these raw materials: methylammonium chlorine $\left(\mathrm{CH}_{3} \mathrm{NH}_{3} \mathrm{Cl}, 99.5 \%\right.$, Advanced Election Technology Co., Ltd), methylammonium bromide $\left(\mathrm{CH}_{3} \mathrm{NH}_{3} \mathrm{Br}\right.$, 99.5\%, Advanced Election Technology Co., Ltd ), lead chlorine $\left(\mathrm{PbCl}_{2}, 99.99 \%\right.$, Advanced Election Technology Co., Ltd ), lead bromide $\left(\mathrm{PbBr}_{2}, 99.9 \%\right.$, Advanced Election Technology Co., Ltd ), N, Ndimethylformamide (DMF, 99\%, Energy Chemical), and dimethylsulfoxide (DMSO, 99\%, Energy Chemical).

In order to make large enough crystals for flexoelectric characterization, we developed an inverse temperature crystallization recipe as shown in Figure $\mathbf{S 1}$. $2.0 \mathrm{M} \mathrm{PbCl}_{2}$ and $2.0 \mathrm{M} \mathrm{CH}_{3} \mathrm{NH}_{3} \mathrm{Cl}$ were dissolved in DMSO-DMF (7:3 by volume) in a vial to keep the molar ratio of $\mathrm{PbCl}_{2}$ to $\mathrm{CH}_{3} \mathrm{NH}_{3} \mathrm{Cl}$ as 1. Later, $1.1 \mathrm{M} \mathrm{CH}_{3} \mathrm{NH}_{3} \mathrm{PbBr}_{3}$ solution in DMF solution was prepared by dissolving equimolar amounts of $\mathrm{CH}_{3} \mathrm{NH}_{3} \mathrm{Br}$ and $\mathrm{PbBr}_{2}$. These precursor solutions were stirred to fully dissolve for $2 \mathrm{~h}$. Subsequently, they were filtered using PTFE filter before heated on an oil bath pan. Small perovskite crystals (average size about $1.5 \mathrm{~mm}$ ) were synthesized as seed crystals in advance by keeping $5 \mathrm{ml}$ of precursor solution at $70^{\circ} \mathrm{C}$ for $5 \mathrm{~h}$. By placing a seed crystal with $\mathrm{U}$ groove in $15 \mathrm{ml}$ precursor solution and gradually increasing the temperature from $60{ }^{\circ} \mathrm{C}$ to $100{ }^{\circ} \mathrm{C}$ for 5 days at the heating rate $0.2-0.5^{\circ} \mathrm{C} / \mathrm{h}$, the seed crystal grew into a larger elongated single crystal (about $15 \mathrm{~mm}$ in length, $5 \mathrm{~mm}$ in width, $1.5 \mathrm{~mm}$ in thickness). 
Growth temperature plays a key role in determining the quality of the final single crystals. In the precursor solution system, the crystal growth rate is dominated by the deposition rate of the solute molecule onto the seed crystal surface and the diffusion rate of the solute in the solution. ${ }^{\mathrm{S1,52}}$ The transparent perfect crystals are obtained in the appropriate temperature which the deposition rate slightly bigger than the diffusion rate. Increasing the temperature from $60^{\circ} \mathrm{C}$ to $100{ }^{\circ} \mathrm{C}$ for 5 days at the heating rate $0.2-0.5^{\circ} \mathrm{C} / \mathrm{h}$ is crucial for the growth of seed crystal. The crystal structure was identified by XRD measurements performed with a PANalytical $X^{\prime}$ pert diffractometer in the Bragg-Brentano parafocusing geometry. As shown in Figure S2, the samples display a pure cubic phase with the $\{$ h00 $\}$ cubic planes parallel to the surfaces of the crystal.

\section{Electrodes}

The Au and AZO-based electrodes were deposited by pulsed laser deposition (PLD), using pure gold metal and $\mathrm{AZO}\left(98 \% \mathrm{ZnO}\right.$ and $2 \% \mathrm{Al}_{2} \mathrm{O}_{3}$ ) targets. Since the $\mathrm{MAPbBr}_{3}$ and $\mathrm{MAPbCl}_{3}$ single crystals cannot resist temperatures higher than $257 \stackrel{\circ}{ } \mathrm{C}$ and $214 \stackrel{\circ}{\circ}$, respectively, ${ }^{S 1}$ the deposition temperature is strictly limited to $150 \stackrel{\circ}{ } \mathrm{C}$. Transparent conductive electrodes were made in the form of $A Z O(100 \mathrm{~nm}) / \mathrm{Au}(10 \mathrm{~nm}) / \mathrm{AZO}(100 \mathrm{~nm})$ sandwitch structures (oxide/metal/oxide, OMO). A KrF excimer laser (COMPexPro205, Coherent) with a wavelength of $248 \mathrm{~nm}$ and pulse duration of $30 \mathrm{~ns}$ delivered an energy of about $220 \mathrm{~mJ}$ per pulse. The laser was operated at $5 \mathrm{~Hz}$, while the target-substrate distance was fixed at $6 \mathrm{~cm}$. The base pressure was set as 4.5 $\mathrm{Pa}$ for $\mathrm{AZO}$ and $8 \times 10^{-5} \mathrm{~Pa}$ for Au films. The deposition temperature for the undercoat $A Z O$ layer was set as 150

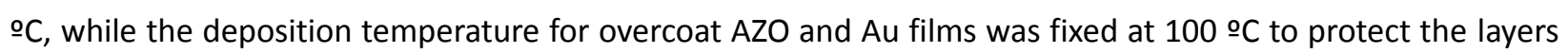
underneath. The AZO/Au/AZO thin film was post-annealed at $100 \stackrel{\circ}{\circ}$ for 30 min with the base pressure of $8 \times 10^{-}$

${ }^{5} \mathrm{~Pa}$. In order to compare and analyze the optical transmittance and electric properties, the AZO/Au/AZO electrode was also deposited on glass and mica substrates by using the above-mentioned process.

The sheet resistance of the electrodes was measured using a four-point-probe tester (RTS-9). The sheet resistance of the transparent electrodes on different substrates is between 8 and $14 \Omega / s q$, sufficiently low for electrical measurements. The sheet resistance of pure gold electrodes was $\sim 0.5 \Omega / \mathrm{sq}$.

\section{Optical characterization}

The optical transmittance of the transparent electrodes and absorption spectra of the perovskite single crystals were measured in the wavelength range of 300 to $800 \mathrm{~nm}$ using an UV spectrometer (SpectraPro-275, Acton Research Corporation) and are shown in Figure S3. The average transmittance of the transparent electrodes on traditional glass and mica substrates is about $75 \%$ in the visible range, and it drops below $30 \%$ for wavelengths below 350nm in the UV range. The transparent electrodes are therefore suitable for photoflexoelectric studies in the visible and near-UV range. Meanwhile, the $\mathrm{MAPbX}_{3}$ crystals have absorption edges around $431 \mathrm{~nm}$ for $\mathrm{MAPbCl}_{3}$ and $574 \mathrm{~nm}$ for $\mathrm{MAPbBr}_{3}$.

\section{Dielectric characterization}

The dielectric properties of $\mathrm{MAPbX}_{3}$ single crystals were measured by a broadband frequency analyzer 
(alpha-A, Novocontrol, Germany) in the frequency range of $10^{-1}-10^{7} \mathrm{~Hz}$. The temperature dependence of the dielectric permittivity and dielectric loss of $\mathrm{MAPbX}_{3}$ single crystals are shown in Figure $\mathrm{S} 4$. Both the $\mathrm{MAPbCl}_{3}$ and $\mathrm{MAPbBr}_{3}$ crystals show a relative high permittivity from room temperature to $140{ }^{\circ} \mathrm{C}$; however, at frequencies lower than $10 \mathrm{kHz}$ there is also a high dielectric loss, indicating leakage contributions consistent with the semiconductor nature of these kind of materials. The temperature and frequency dependence of the capacitance and losses are typical of a barrier layer capacitor (Maxwell-Wagner behavior).

\section{Photoflexoelectric and Photopiezoelectric Characterization.}

The flexoelectric setup is shown in Figure S5. A clamped-cantilever system was employed for measuring the flexoelectric coefficient of the crystals. The free end of the crystal cantilever was driven by a piezoelectric actuator fixed to three-dimension mobile platform. A signal generator (Tektronix AFG3101C) provides an $\mathrm{AC}$ voltage for the actuator. We measured at different oscillation frequencies from $0.3 \mathrm{H}$ to 120 $\mathrm{Hz}$ (figure 3-a). For fixed-frequency measurements such as flexoelectricity vs temperature or flexoelectricity vs deflection amplitude, we measured at $7 \mathrm{~Hz}$, a prime-number chosen to avoid any potential ringing interference from main power lines.

The bending-induced currents were measured using a lock-in amplifier (Stanford Research SR830 DSP) and converted to charge density by dividing the measured current I by the driving frequency and the area of the electrodes.

$$
P=\frac{I}{\omega A}
$$

where $A$ is the area of the electrodes and $\omega=2 \pi v$ is the angular frequency of the mechanical oscillation. An oscilloscope (Tektronix TDS2012C) was used for real-time monitoring of the generated signals. To measure the temperature-dependence of flexoelectric coefficients, we used a vacuum oven with a transparent lid and watch window was employed. The temperature control resolution of the oven is 0.5 ${ }^{\circ} \mathrm{C}$, and the operation temperature ranges from room temperature to $200^{\circ} \mathrm{C}$.

For the photo-flexoelectric measurement, we integrated an UV lamp (Lesfa YG) with adjustable power on the float table. The ultraviolet or white light was illuminated perpendicularly to the transparent electrode layer. When using gold electrodes, we illuminated from the side instead (Figure S6). The absorbed light intensity of the crystal was measured by a photodetector (Lihuada DT1334A). These experiments were performed with the rest of the laboratory in the dark.

The polarization is calculated from the current using (3), and the effective flexoelectric coefficient is defined as the induced polarization divided by the average strain gradient $\frac{\overline{\partial S_{11}}}{\partial x_{3}}$ :

$$
\mu_{\text {eff }}=\frac{P}{\overline{\frac{\partial S_{11}}{\partial x_{3}}}}=\frac{I}{\omega A \frac{\partial S_{11}}{\partial x_{3}}}
$$

The strain gradient was calculated from the elastic equation for the vertical deformation ( $y$ ) of a point- 
loaded, single clamp cantilever. The deformation can be considered as quasi-static because our measurement frequency $(7 \mathrm{~Hz})$ is several orders of magnitude below the resonance frequency range of the cantilevers (tens of $\mathrm{kHz}$ ). The clamped beam deformation is:

$$
y(x)=\frac{\delta x^{2}}{2 L^{3}}(3 L-x)
$$

where $\delta$ is the vertical deflection delivered by the piezoelectric actuator at the end of the cantilever, $L$ is the length of the cantilever, and $x$ is the horizontal position along the cantilever, with the origin at the clamping point. The strain gradient is the curvature of the deflection function, which is the second derivative with respect to $x$ :

$$
\frac{\partial S_{11}}{\partial x_{3}}=\frac{\partial^{2} y(x)}{\partial x^{2}}=\frac{3 \delta}{L^{3}}(L-x)
$$

The average curvature under the electrode is therefore:

$$
\overline{\frac{\partial S_{11}}{\partial x_{3}}}=\frac{3}{2} \frac{\delta}{L^{2}}
$$

\section{Uniaxial compression experiments.}

To ensure that the current is due to strain gradient (flexoelectricity) and not plain strain (piezoelectricity), we modified the deformation setup, placing the sample on a flat surface and adding a piece of transparent glass between the actuator and the sample so as to distribute the mechanical load. In Figure S7 we show the modified setup, together with the comparison between the results for bendinginduced charge and compression-induced charge for the same mechanical load. While oscillatory bending causes an alternate current, periodic compression causes no electrical response at all. Piezoelectricity is thereby excluded.

In contrast, when the top and bottom surfaces of a crystal are of different size, uniaxial compression results in a vertical pressure gradient (the compression force is distributed over different areas at the top and bottom, pressure being bigger on the side that has a smaller area). This stress difference causes a vertical strain gradient that can generate flexoelectricity. ${ }^{[512,513]}$ For trapezoid-shaped crystals, the strain gradient is

$$
\frac{\partial S_{33}}{\partial z}=\frac{\frac{F}{c_{11} a_{2} b}-\frac{F}{c_{11} a_{1} b}}{h}
$$

Where $F$ is the applied force, $c_{11}$ is the elastic constant, $a_{1}$ and $a_{2}$ are top and bottom length dimensions of the trapezoidal table, respectively, $b$ is the width dimension, $h$ is the thickness dimension of the trapezoidal table (see Figure S8). As in the bending experiment, the charge was measured under oscillatory load with a lock-in amplifier, and divided by the strain-gradient in order to obtain the effective flexoelectric coefficient. 


\section{Data availability}

The data used for the plots in the article are provided as a Word file in the supplementary material. Other datasets generated and/or analyzed during the current study are available from L. Shu (llshu@ncu.edu.cn) on reasonable request.

\section{Acknowledgements}

This work was supported by the National Natural Science Foundation of China under Grant Nos. 51962020, 51972157,11574126 and 11604135 , and partly by the National Key Research and Development Plan of China (2017YFB0406300). L. Shu and S. Ke thanks the support from Nanchang University. G. Catalan acknowledges support from the Generalitat de Catalunya (Grant 2017 SGR 579), and from MINECO (National Plan MAT201677100-C2-1-P and Severo Ochoa SEV-2017-0706).

\section{Contributions}

G. Catalan and L. Shu conceived the idea and coordinated this work. M. Stengel produced the theoretical model. G. Catalan wrote the paper. L. Shu, S. Ke, L. Fei, W. Huang, Z. Wang, J. Gong prepared the samples and performed the photoflexoelectric experiments., X. Jiang, L. Wang, S. Lei, F. Li, Z. Rao, R. Zheng, X. Yao, Y. Zhou, Y. Wang did the other experimental measurements and joined the discussions.

\section{Competing interests}

The authors declare no competing financial interests. 